\title{
Stability Analysis of an In-Host Viral Model with Cure of Infected Cells and Humoral Immunity
}

\author{
Hui Wang, Rong Wang, Zhixing Hu, and Fucheng Liao \\ Department of Applied Mathematics, University of Science and Technology Beijing, Beijing 100083, China \\ Correspondence should be addressed to Hui Wang; bkdwh@163.com
}

Received 29 August 2013; Accepted 4 December 2013

Academic Editor: Junjie Wei

Copyright ( 2013 Hui Wang et al. This is an open access article distributed under the Creative Commons Attribution License, which permits unrestricted use, distribution, and reproduction in any medium, provided the original work is properly cited.

\begin{abstract}
An in-host viral model with cure of infected cells and humoral immunity is studied. We prove that the stability is completely determined by the basic reproductive number $R_{0}$ and show that the infection-free equilibrium $E_{0}$ is globally asymptotically stable if and only if $R_{0} \leq 1$. Moreover, if $R_{0}>1$, the infection equilibrium is locally asymptotically stable when the time delay $\tau$ is small and it loses stability as the length of the time delay increases past a critical value $\tau_{0}$. Finally, we confirm our analysis by providing several numerical examples.
\end{abstract}

\section{Introduction}

The humoral immunity is a kind of immunologic mechanism which uses B lymphocytes to produce antigen to prevent virus and there are evidences to prove that the humoral immunity is more effective than the cell-mediated immune in some infections such as malaria infection [1-3]. Many authors present and develop mathematical systems for the humoral immunity [4-7]. And the cure of virus is also important especially in HBV models $[8,9]$. In the present paper, we analyze an in-host viral model with humoral immunity and intracellular delay, and we incorporate a "cure" of infected cells into it. We propose the following system:

$$
\begin{gathered}
T^{\prime}(t)=\Lambda-\beta T(t) V(t)-d T(t)+b I(t), \\
I^{\prime}(t)=\beta T(t) V(t)-(a+b) I(t), \\
V^{\prime}(t)=k I(t)-u V(t)-q B(t) V(t), \\
B^{\prime}(t)=g V(t-\tau)-c B(t),
\end{gathered}
$$

where $T, I, V$, and $B$ represent the uninfected cells, the infected cells, the virus, and the B cells, respectively. $\Lambda$ and $d$ are assumed as the birth rate and death rate of uninfected cells. $\beta$ is the infection rate and $k I(t)$ represents the number of free virus which is produced during the average infected cell life span. $a$ is the death rate of infected cells and $u$ represents the death rate of virus. $g$ and $c$ represent the birth rate and death rate of $\mathrm{B}$ cells. The $\mathrm{B}$ cells neutralization rate is represented by $q$.

The following form is taken as the initial conditions:

$$
\begin{gathered}
T(\theta)=\varphi_{1}(\theta), \\
I(\theta)=\varphi_{2}(\theta), \\
V(\theta)=\varphi_{3}(\theta), \\
B(\theta)=\varphi_{4}(\theta) \\
\varphi_{i}(\theta) \geq 0, \quad \theta \in(-\tau, 0), \quad \varphi_{i}(0)>0 \quad(i=1,2,3,4),
\end{gathered}
$$

where $\left(\varphi_{1}(\theta), \varphi_{2}(\theta), \varphi_{3}(\theta), \varphi_{4}(\theta)\right) \in \mathbb{C}\left([-\tau, 0], \mathbb{R}_{+0}^{4}\right)$, the space of continuous functions mapping the interval $(-\infty, 0]$ into $\mathbb{R}_{+0}^{4}$, and

$$
\mathbb{R}_{+0}^{4}=\left\{\left(x_{1}, x_{2}, x_{3}, x_{4}\right) \mid x_{i} \geq 0, i=1,2,3,4\right\} .
$$

The organization of this paper is as follows. In the next section, we will find threshold parameters $R_{0}$ of system (1) and it determines the existence of the equilibriums. In Section 3, by structuring suitable Lyapunov functionals and using LaSalle's invariance principle we attain the global stability of the uninfected equilibrium if $R_{0} \leq 1$. In Section 4 , we consider the stability of the infected equilibrium and 
the occurrence of local Hopf bifurcation. In Section 5, we present the numerical simulations to illustrate our results. Finally, we offer concluding remarks in the last section.

\section{Existence of Equilibrium}

We can easily find that system (1) always has an uninfected equilibrium $E_{0}=\left(T_{0}, 0,0,0\right)=((\Lambda / d), 0,0,0)$.

Denote

$$
R_{0}=\frac{\Lambda k \beta}{u d(a+b)} .
$$

We call $R_{0}$ the basic reproductive number. It is easy to prove that if $R_{0}>1$, model (1) exist an infected equilibrium $E^{*}\left(T^{*}, I^{*}, V^{*}, B^{*}\right)$, where

$$
\begin{gathered}
V^{*}=\left(\sqrt{(\beta c u a-(a+b) d g q)^{2}+4 \Lambda g q a c k \beta^{2}}\right. \\
-\beta c u a-(a+b) d g q) \\
\times(2 a \beta g q)^{-1}, \\
T^{*}=\frac{g q(a+b)}{k \beta c} V^{*}+\frac{u(a+b)}{k \beta}, \\
B^{*}=\frac{g}{c} V^{*}, \\
I^{*}=\frac{g q}{k c} V^{* 2}+\frac{u}{k} V^{*} .
\end{gathered}
$$

\section{Global Attraction of Infection-Free Equilibrium}

Theorem 1. The infection-free equilibrium $E_{0}$ of system (1) is globally attractive if $R_{0} \leq 1$.

Proof. Let $(T(t), I(t), V(t), B(t))$ be any positive solution of system (1) with initial conditions (2).

Define

$$
\begin{aligned}
L(t)= & \frac{g k}{2 q(a+b)} \cdot \frac{\left(T-T_{0}\right)^{2}}{T_{0}} \\
& +\frac{b g k}{2 q(a+b)(a+d)} \cdot \frac{\left(T-T_{0}+I\right)^{2}}{T_{0}} \\
& +\frac{g k}{q(a+b)} I+\frac{g}{q} V+\frac{1}{2} B^{2} \\
& +g B \int_{t-\tau}^{t} V(\xi) d \xi .
\end{aligned}
$$

Calculating the derivative of $L(t)$ along positive solutions of system (1) and noting $\Lambda=d T_{0}$ and $T V\left(T-T_{0}\right)=V\left(T-T_{0}\right)^{2}+$ $T_{0} V\left(T-T_{0}\right)$, it follows that

$$
\begin{aligned}
\dot{L}= & -\left(\frac{g k \beta}{q(a+b)} V+\frac{d g k(a+d)+b d g k}{q(a+b)(a+d)}\right) \\
& \times \frac{\left(T-T_{0}\right)^{2}}{T_{0}}-\frac{a b g k}{q(a+b)(a+d)} I^{2} \\
& -c B^{2}+\frac{g u}{q}\left(R_{0}-1\right) V .
\end{aligned}
$$

If $R_{0} \leq 1$, it follows from (7) that $\dot{L} \leq 0$. The solutions are limited to the largest invariant subset $M$ of $\{\dot{L}(t)=0\}$. It is clear that $\dot{L}(t)=0$ if and only if $(T, I, V, B)=\left(T_{0}, I_{0}, V_{0}, B_{0}\right)$. So we can obtain the global attraction of $E_{0}$ by using LaSalle's invariance principle. This completes the proof.

\section{Permanence of the System for $R_{0}>1$}

Theorem 2. If $R_{0}>1$, the infected equilibrium $E^{*}$ of system (1) is locally asymptotically stable when $\tau=0$.

Proof. The characteristic equation associated with system (1) at $E^{*}$ is

$$
\begin{aligned}
\lambda^{4}+ & m_{3} \lambda^{3}+m_{2} \lambda^{2} \\
& +m_{1} \lambda+m_{0}+e^{-\lambda \tau}\left(n_{2} \lambda^{2}+n_{1} \lambda+n_{0}\right)=0 .
\end{aligned}
$$

Noting that $T^{*}=((g q(a+b)) /(k \beta c)) V^{*}+((u(a+b)) /(k \beta))$, $B^{*}=(g / c) V^{*}$, we can obtain

$$
\begin{gathered}
m_{3}=a+b+c+d+u+q B^{*}+\beta V^{*}, \\
m_{2}=b d+a\left(d+\beta V^{*}\right) \\
+\left(u+q B^{*}\right)\left(d+\beta V^{*}\right) \\
+c\left(a+b+d+u+q B^{*}+\beta V^{*}\right), \\
m_{1}=\frac{(a+c) g q}{c} \beta V^{* 2} \\
+(a u+c u+a c) \beta V^{*}+c d(a+u+b), \\
m_{0}=c\left(a u \beta V^{*}+a q \beta V^{*} B^{*}\right), \\
n_{2}=g q V^{*}, \\
n_{1}=g q V^{*}\left(a+b+d+\beta V^{*}\right), \\
n_{0}=d g q(a+b) V^{*}+a g q \beta V^{* 2} .
\end{gathered}
$$

If $\tau=0$, (8) becomes

$$
\lambda^{4}+m_{3} \lambda^{3}+\left(m_{2}+n_{2}\right) \lambda^{2}+\left(m_{1}+n_{1}\right) \lambda+m_{0}+n_{0}=0 .
$$

Then we can obtain $m_{0}+n_{0}>0, m_{1}+n_{1}>0, m_{2}+n_{2}>0$, and $m_{3}>0$. It follows from Routh-Hurwitz criterion that all roots of (10) have negative real parts when $\tau=0$. This competes the proof. 
Let $\lambda=i v$ and then substitute it into (8). Separating the real and imaginary parts, we will gain

$$
\begin{gathered}
v^{4}-m_{2} v^{2}+m_{0}=\left(n_{2} v^{2}-n_{0}\right) \cos v \tau-n_{1} v \sin v \tau, \\
m_{3} v^{3}-m_{1} v=\left(n_{2} v^{2}-n_{0}\right) \sin v \tau+n_{1} v \cos v \tau .
\end{gathered}
$$

Squaring and adding (11) yield

$$
\begin{aligned}
v^{8}+ & \left(m_{3}^{2}-2 m_{2}\right) v^{6} \\
& +\left(m_{2}^{2}-2 m_{1} m_{3}+2 m_{0}-n_{2}^{2}\right) v^{4} \\
& +\left(m_{1}^{2}-2 m_{0} m_{2}-n_{1}^{2}+2 n_{0} n_{2}\right) v^{2} \\
& +\left(m_{0}^{2}-n_{0}^{2}\right)=0 .
\end{aligned}
$$

Denote

$$
\begin{aligned}
G(x)= & x^{4}+\left(m_{3}^{2}-2 m_{2}\right) x^{3} \\
& +\left(m_{2}^{2}-2 m_{1} m_{3}+2 m_{0}-n_{2}^{2}\right) x^{2} \\
& +\left(m_{1}^{2}-2 m_{0} m_{2}-n_{1}^{2}+2 n_{0} n_{2}\right) x \\
& +\left(m_{0}^{2}-n_{0}^{2}\right) .
\end{aligned}
$$

We have that

$$
\begin{aligned}
G^{\prime}(x)= & 4 x^{3}+3\left(m_{3}^{2}-2 m_{2}\right) x^{2} \\
& +2\left(m_{2}^{2}-2 m_{1} m_{3}+2 m_{0}-n_{2}^{2}\right) x \\
& +\left(m_{1}^{2}-2 m_{0} m_{2}-n_{1}^{2}+2 n_{0} n_{2}\right) .
\end{aligned}
$$

Hence if $G(x)=0$ has a positive $\operatorname{root} x=v^{2}$, then $\lambda= \pm i v$ are a couple of purely imaginary characteristic roots. Moreover, for a real practice model with fully known coefficients, the exactly four roots of $G(x)=0$ can be numerically calculated with the help of computational software such as Matlab. Let $x_{i}(1 \leq i \leq k, 1 \leq k \leq 4)$ be the positive roots of $G(x)=0$ and $v_{i}=\sqrt{x_{i}}$.

Solving (11) with respect to $\tau$, we can obtain

$$
\begin{aligned}
& \tau_{i}^{(j)}=\frac{1}{v_{i}} \arccos \left(\left(\frac{n_{2} v_{i}^{6}+\left(-n_{0}+n_{1} m_{3}-n_{2} m_{2}\right) v_{i}^{4}}{n_{1}^{2} v_{i}^{2}+\left(n_{2} v_{i}^{2}-n_{0}\right)^{2}}\right)\right. \\
&\left.+\left(\frac{\left(n_{0} m_{2}-n_{1} m_{1}+n_{2} m_{0}\right) v_{i}^{2}-n_{0} m_{0}}{n_{1}^{2} v_{i}^{2}+\left(n_{2} v_{i}^{2}-n_{0}\right)^{2}}\right)\right) \\
&+\frac{1}{v_{i}} \cdot 2 j \pi,
\end{aligned}
$$

where $1 \leq i \leq k ; 1 \leq k \leq 4 ; j=0,1,2, \ldots$. Let

$$
\tau_{0}=\min \left\{\tau_{i}^{(j)} \mid 1 \leq i \leq k ; j=0,1,2, \ldots\right\} .
$$

This means that $\tau_{0}$ is the least value of $\tau$ which can be used to make the characteristic equation have purely imaginary roots.

Theorem 3. $G^{\prime}\left(v_{i}^{2}\right)$ and $\operatorname{Re}[d \lambda / d \tau]_{\tau=\tau_{i}^{(j)}}^{-1}$ have the same sign.
Proof. Let the characteristic equation be in the following form:

$$
f(\lambda)+g(\lambda) e^{-\lambda \tau}=0 .
$$

We can obviously know from (8) that

$$
\begin{gathered}
f(\lambda)=\lambda^{4}+m_{3} \lambda^{3}+m_{2} \lambda^{2}+m_{1} \lambda+m_{0}, \\
g(\lambda)=n_{2} \lambda^{2}+n_{1} \lambda+n_{0} .
\end{gathered}
$$

Calculating the derivative of (17) with respect to $\tau$, we can obtain that

$$
\begin{gathered}
\frac{d \lambda}{d \tau}\left(f^{\prime}(\lambda)+g^{\prime}(\lambda) e^{-\lambda \tau}-g(\lambda) e^{-\lambda \tau} \cdot \tau\right) \\
\quad=g(\lambda) e^{-\lambda \tau} \cdot \lambda,
\end{gathered}
$$

$$
\begin{aligned}
{\left[\frac{d \lambda}{d \tau}\right]_{\tau=\tau_{i}^{(j)}}^{-1} } & =\frac{f^{\prime}(\lambda)+g^{\prime}(\lambda) e^{-\lambda \tau}}{g(\lambda) \mathrm{e}^{-\lambda \tau} \cdot \lambda}-\frac{\tau}{\lambda} \\
& =\frac{f^{\prime}\left(i v_{i}\right)+g^{\prime}\left(i v_{i}\right) e^{-i v_{i} \tau}}{g\left(i v_{i}\right) e^{-i v_{i} \tau} \cdot i v_{i}}-\frac{\tau}{i v_{i}} .
\end{aligned}
$$

Therefore,

$$
\begin{aligned}
\operatorname{Re}\left[\frac{d \lambda}{d \tau}\right]_{\tau=\tau_{i}^{(j)}}^{-1} \\
=\operatorname{Re}\left[\frac{f^{\prime}\left(i v_{i}\right)+g^{\prime}\left(i v_{i}\right) e^{-i v_{i} \tau}}{g\left(i v_{i}\right) e^{-i v_{i} \tau} \cdot i v_{i}}\right] \\
=\operatorname{Re}\left[\frac{f^{\prime}\left(i v_{i}\right)}{i v_{i} \cdot\left(-f\left(i v_{i}\right)\right)}+\frac{g^{\prime}\left(i v_{i}\right)}{i v_{i} \cdot g\left(i v_{i}\right)}\right] \\
=\operatorname{Re}\left[\frac{1}{i v_{i}} \cdot\left(\frac{-f^{\prime}\left(i v_{i}\right) \cdot \bar{f}\left(i v_{i}\right)}{f\left(i v_{i}\right) \cdot \bar{f}\left(i v_{i}\right)}+\frac{g^{\prime}\left(i v_{i}\right) \cdot \bar{g}\left(i v_{i}\right)}{g\left(i v_{i}\right) \cdot \bar{g}\left(i v_{i}\right)}\right)\right] \\
=\operatorname{Re}\left[\frac{1}{i v_{i}} \cdot \frac{g^{\prime}\left(i v_{i}\right) \cdot \bar{g}\left(i v_{i}\right)-f^{\prime}\left(i v_{i}\right) \cdot \bar{f}\left(i v_{i}\right)}{\left|f\left(i v_{i}\right)\right|^{2}}\right] .
\end{aligned}
$$

Obviously,

$$
\begin{aligned}
\operatorname{sign}\left(\operatorname{Re}\left[\frac{d \lambda}{d \tau}\right]_{\tau=\tau_{i}^{(j)}}^{-1}\right) \\
\quad=\operatorname{sign}\left(\operatorname{Im}\left(g^{\prime}\left(i v_{i}\right) \cdot \bar{g}\left(i v_{i}\right)-f^{\prime}\left(i v_{i}\right) \cdot \bar{f}\left(i v_{i}\right)\right)\right) .
\end{aligned}
$$

And after some calculations, we can get

$$
\begin{aligned}
& \operatorname{Im}\left(g^{\prime}\left(i v_{i}\right) \cdot \bar{g}\left(i v_{i}\right)-f^{\prime}\left(i v_{i}\right) \cdot \bar{f}\left(i v_{i}\right)\right) \\
&= 4 v_{i}^{7}+3\left(-2 m_{2}+m_{3}^{2}\right) v_{i}^{5} \\
&+2 \cdot\left(m_{2}^{2}-2 m_{1} m_{3}+2 m_{0}-n_{2}^{2}\right) v_{i}^{3} \\
&+\left(m_{1}^{2}-2 m_{0} m_{2}-n_{1}^{2}+2 n_{0} n_{2}\right) v_{i} .
\end{aligned}
$$




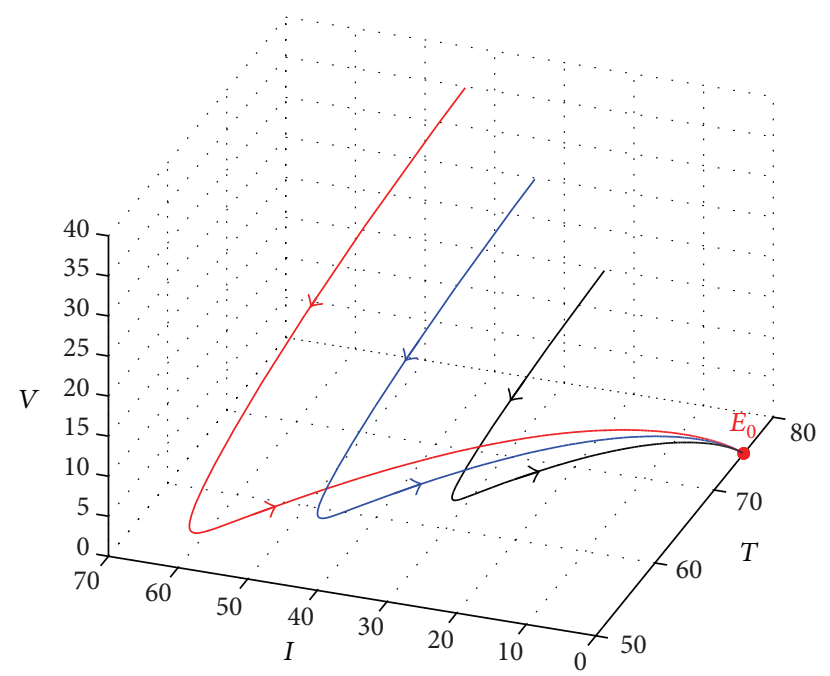

FIGURE 1: Equilibrium $E_{0}$ is globally asymptotically stable if $R_{0}<1$.

Therefore, $G^{\prime}\left(v_{i}^{2}\right)$ and $\operatorname{Re}[d \lambda / d \tau]_{\tau=\tau_{i}^{(j)}}^{-1}$ have the same sign. This completes the proof.

Applying Theorems 2 and 3 and the Hopf bifurcation theorem for functional differential equation [10], we derive the existence of a Hopf bifurcation as stated in Theorem 4.

Theorem 4. Suppose that $G(x)=0$ has at least one simple positive root and $\tau_{0}$ is defined in (16). Then there is a Hopf bifurcation for the system (1) as s passes upwards through $\tau_{0}$ leading to a periodic solution that bifurcates from $E^{*}$, where

$$
\begin{aligned}
\tau_{i}^{(j)}=\frac{1}{v_{i}} \arccos ( & \left(\frac{n_{2} v_{i}^{6}+\left(-n_{0}+n_{1} m_{3}-n_{2} m_{2}\right) v_{i}^{4}}{n_{1}^{2} v_{i}^{2}+\left(n_{2} v_{i}^{2}-n_{0}\right)^{2}}\right) \\
& \left.+\left(\frac{\left(n_{0} m_{2}-n_{1} m_{1}+n_{2} m_{0}\right) v_{i}^{2}-n_{0} m_{0}}{n_{1}^{2} v_{i}^{2}+\left(n_{2} v_{i}^{2}-n_{0}\right)^{2}}\right)\right) \\
& +\frac{1}{v_{i}} \cdot 2 j \pi, \quad 1 \leq i \leq k ; \quad j=0,1,2, \ldots
\end{aligned}
$$

\section{Numerical Examples}

Example 1. For system (1), consider all parameters as follows: $a=0.1, b=0.1, \beta=0.1, c=0.001, d=0.2, g=0.1$, $k=0.1, \lambda=1, q=0.001, u=10$, and $\tau=10$. In this case, we can obtain that $R_{0}=0.375<1$; the global attraction of the infection-free equilibrium $E_{0}$ is illustrated by Figure 1.

Example 2. Consider all parameters of system (1) as follows: $a=2.41979, b=2.80472, \beta=2.34292, c=2.2057, d=$ $0.9129, g=4.2074, k=2.6415, \lambda=4.60059, q=3.738$, and $u=1.3978$. In this case, we can obtain that $R_{0}=4.2709>1$.

(1) When $\tau=0.75, E^{*}$ is locally asymptotically stable and the stability is illustrated by Figure 2 .

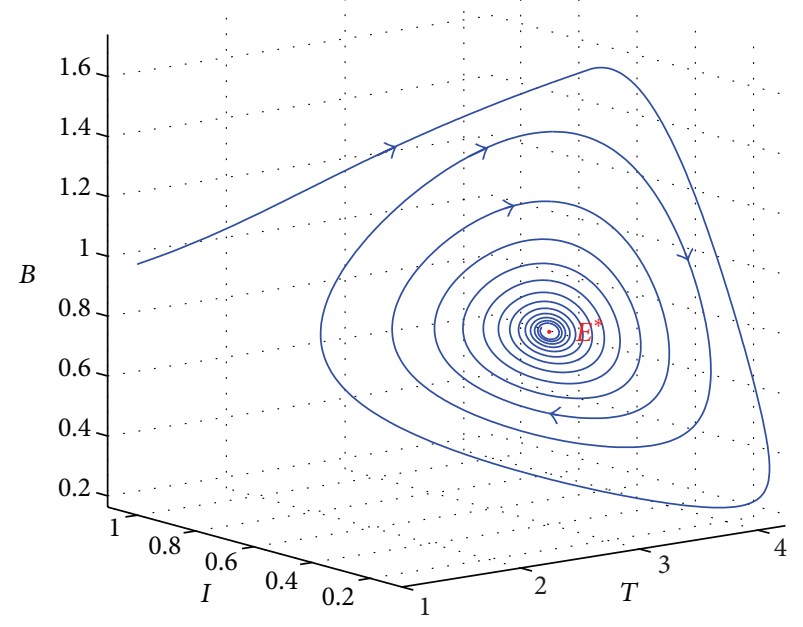

FIGURE 2: Equilibrium $E^{*}$ is locally asymptotically stable when $\tau$ is very small.

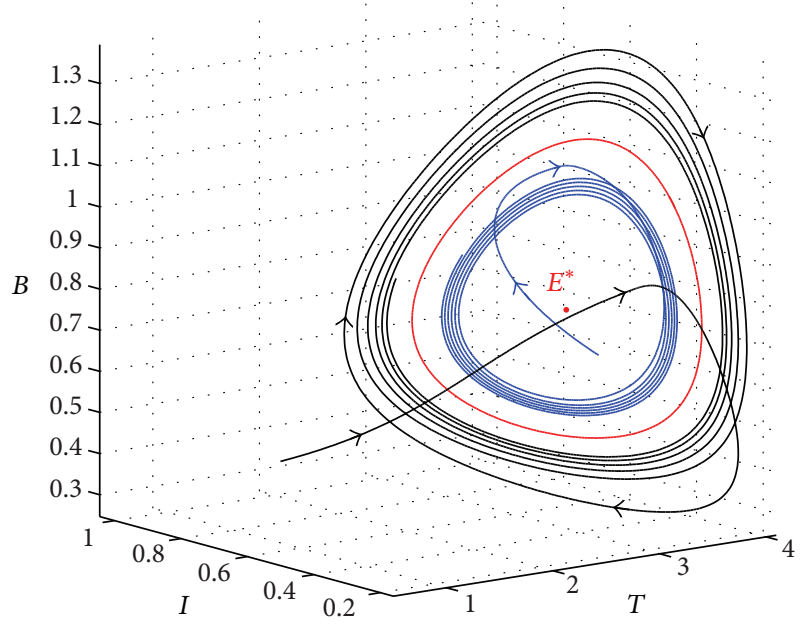

Figure 3: Delay can destabilize $E^{*}$ and lead to Hopf bifurcation when $\tau$ increases past a critical value $\tau_{0}$.

(2) When $\tau=0.9$, the delay can destabilize $E^{*}$ and lead to Hopf bifurcation. We will show that in Figure 3 .

\section{Discussions}

In this paper, we consider an in-host viral model humoral immunity. In addition, a "cure" of infected cells is incorporated. Firstly, it has been shown that if the basic reproductive number is less than unity, the infection-free equilibrium $E_{0}$ is globally attractive, and the time delay has no effect on the dynamics of the system. Then, a detailed analysis on the local asymptotic stability of the infection equilibrium $E^{*}$ of the model is carried out. If $E^{*}$ is feasible, the basic reproduction number of the virus is greater than unity; $E^{*}$ is globally attractive for any time delay under some parameter conditions. By taking the discrete time delay as a bifurcation parameter, it is shown that this system undergoes a sequence 
of Hopf bifurcations and stability switches are observed by using simulations.

\section{Acknowledgments}

This work was supported by National Natural Science Foundation of China (61174209 and 11071013), the Basic Scientific Research Foundation of Central University (FRF-BR-11-048B and FRF-BR-12-004), and the Basic Theory Research Foundation for Engineering Research Institute of USTB (YJ2012$001)$.

\section{References}

[1] M. Nowak and C. Bangham, "Population dynamics of immune responses to persistent viruses," Science, vol. 272, no. 5258, pp. 74-79, 1996.

[2] H. Zhu and X. Zou, "Dynamics of a HIV-1 infection model with cell-mediated immune response and intracellular delay," Discrete and Continuous Dynamical Systems B, vol. 12, no. 2, pp. 511-524, 2009.

[3] X. Wang and Y. Tao, "Lyapunov function and global properties of virus dynamics with CTL immune response," International Journal of Biomathematics, vol. 1, no. 4, pp. 443-448, 2008.

[4] R. M. Anderson, R. M. May, and S. Gupta, "Non-linear phenomena in host-parasite interactions," Parasitology, vol. 99, pp. S59-S79, 1989.

[5] A. Murase, T. Sasaki, and T. Kajiwara, "Stability analysis of pathogen-immune interaction dynamics," Journal of Mathematical Biology, vol. 51, no. 3, pp. 247-267, 2005.

[6] D. Wodarz, R. M. May, and M. A. Nowak, "The role of antigenindependent persistence of memory cytotoxic T lymphocytes," International Immunology, vol. 12, no. 4, pp. 467-477, 2000.

[7] C. Chiyaka, W. Garira, and S. Dube, "Modelling immune response and drug therapy in human malaria infection," Computational and Mathematical Methods in Medicine, vol. 9, no. 2, pp. 143-163, 2008.

[8] C. Vargas-De-León, "Stability analysis of a model for HBV infection with cure of infected cells and intracellular delay," Applied Mathematics and Computation, vol. 219, no. 1, pp. 389398, 2012.

[9] K. Wang, A. Fan, and A. Torres, "Global properties of an improved hepatitis B virus model," Nonlinear Analysis: Real World Applications, vol. 11, no. 4, pp. 3131-3138, 2010.

[10] J. Hale, Theory of Functional Differential Equations, Springer, New York, NY, USA, 1977. 


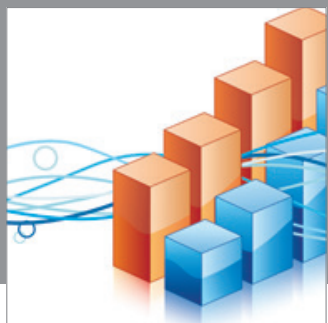

Advances in

Operations Research

mansans

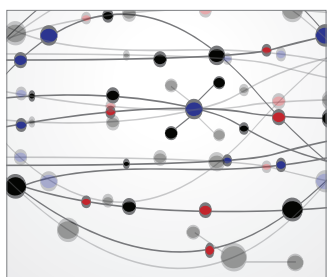

The Scientific World Journal
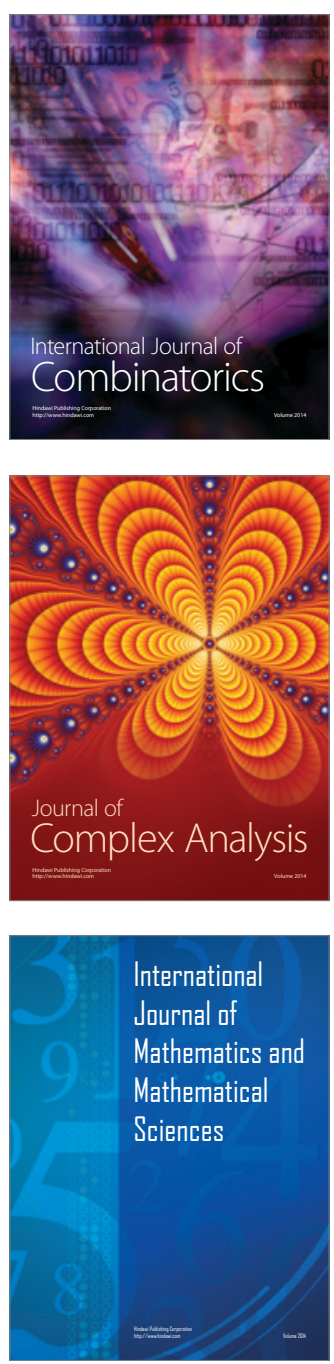
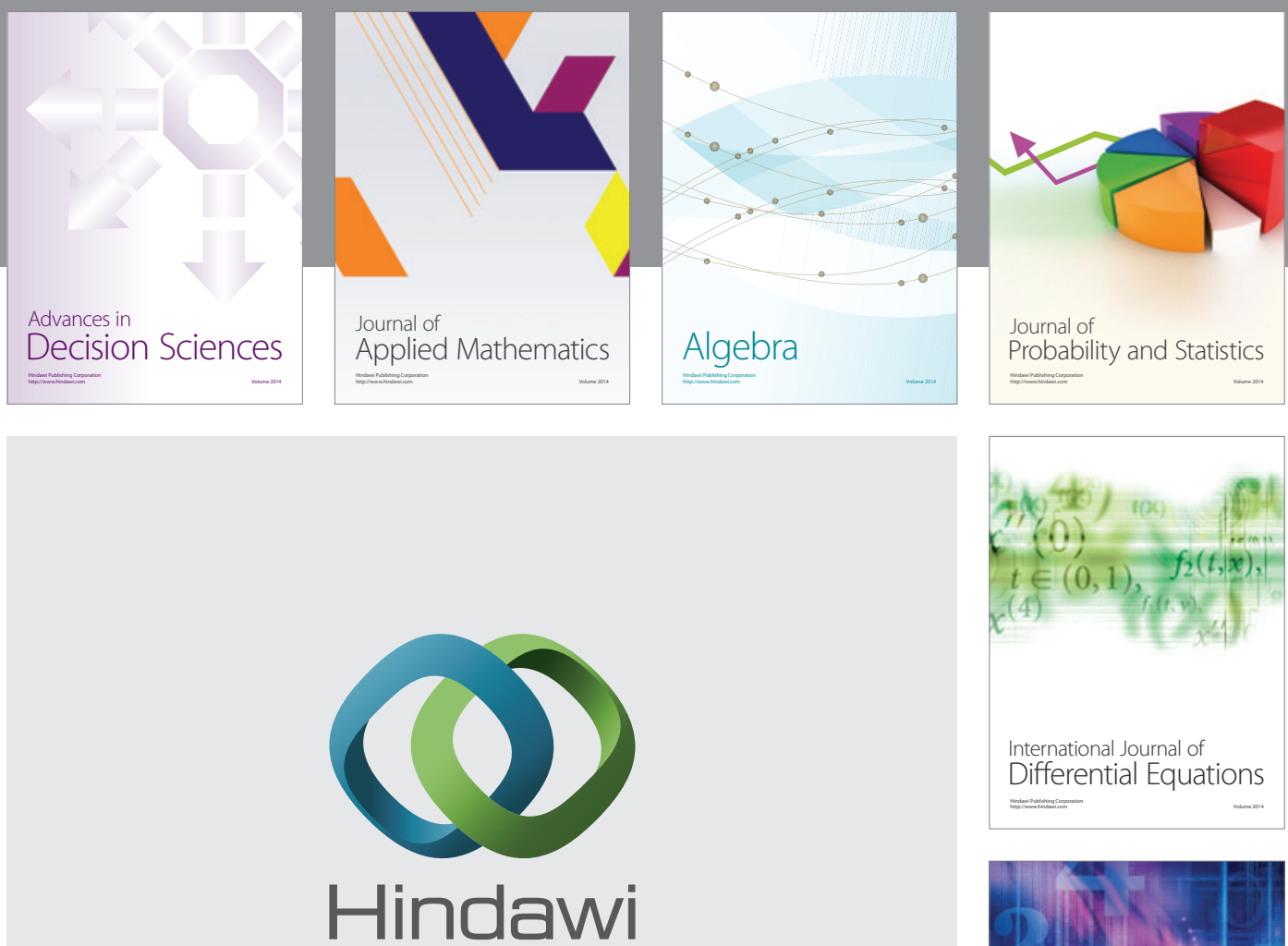

Submit your manuscripts at http://www.hindawi.com
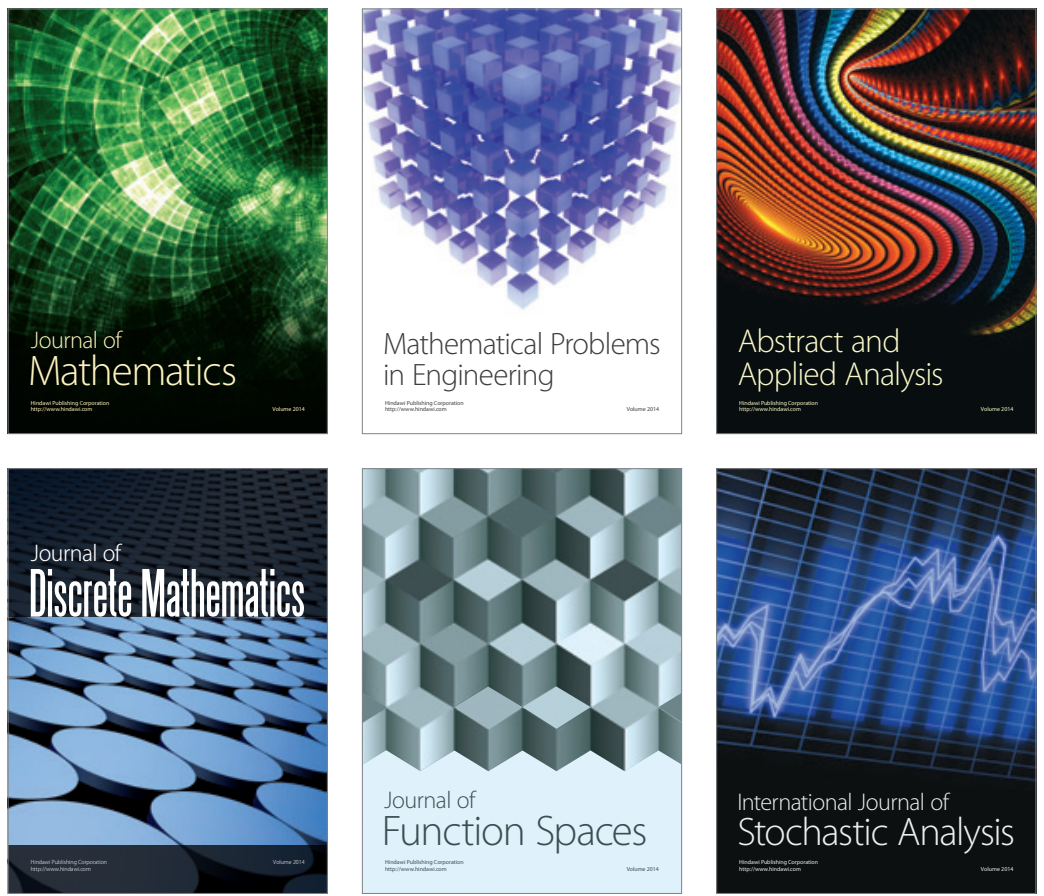

Journal of

Function Spaces

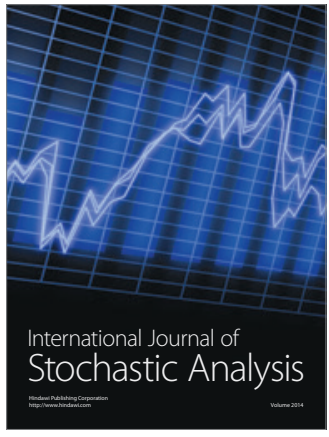

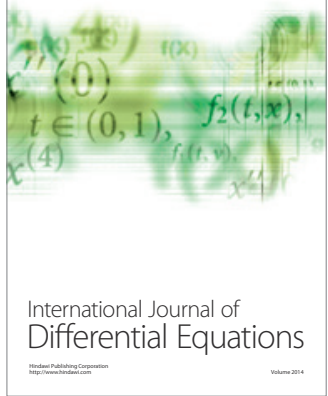
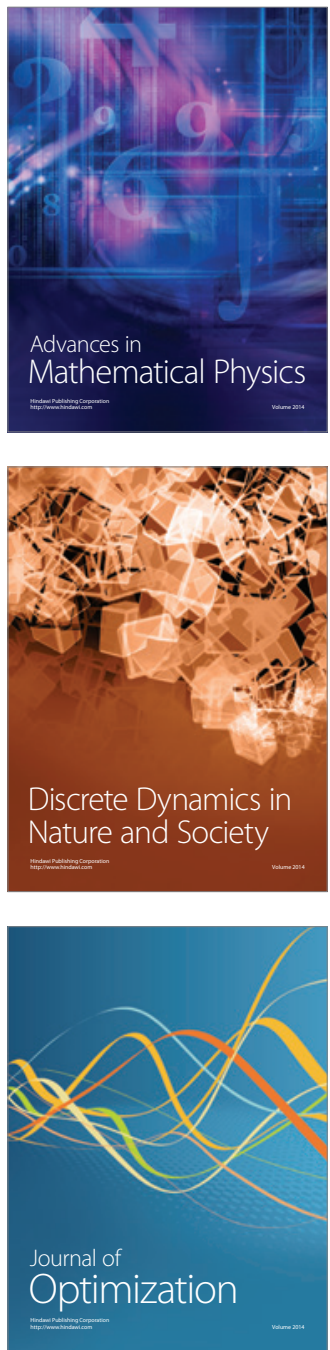